\title{
Long Time Tails in Stationary Random Media. I. Theory
}

\author{
M. H. Ernst, ${ }^{1}$ J. Machta, ${ }^{2}$ J. R. Dorfman, ${ }^{3}$ and H. van Beijeren ${ }^{4}$
}

Received September 2, 1983

\begin{abstract}
Diffusion of moving particles in stationary disordered media is studied using a phenomenological mode-coupling theory. The presence of disorder leads to a generalized diffusion equation with memory kernels having power law long time tails. The velocity autocorrelation function is found to decay like $t^{-(d / 2+1)}$, while the time correlation function associated with the super-Burnett coefficient decays like $t^{-d / 2}$ for long times. The theory is applicable to a wide variety of dynamical and stochastic systems including the Lorentz gas and hopping models. We find new, general expressions for the coefficients of the long time tails which agree with previous results for exactly solvable hopping models and with the low-density results obtained for the Lorentz gas. Finally we mention that if the moving particles are charged, then the long time tails imply that there is an $\omega^{d / 2}$ contribution to the low-frequency part of the frequency-dependent electrical conductivity.
\end{abstract}

KEY WORDS: Diffusion; random media; fluctuations; long time tails; Lorentz model; hopping models; velocity correlation functions; mode coupling theory; diffusion coefficients; Burnett coefficients.

\section{INTRODUCTION}

In this paper we consider some features of the theory of diffusion of particles in a stationary random medium. Our aim is to construct a mode

\footnotetext{
${ }^{1}$ Institute for Theoretical Physics, State University, 3508 TA, Utrecht, The Netherlands.

${ }^{2}$ Department of Physics and Astronomy, University of Massachusetts, Amherst, Massachusetts 01003 .

${ }^{3}$ Institute for Physical Science and Technology and Department of Physics and Astronomy, University of Maryland, College Park, Maryland 20742.

${ }^{4}$ Institute for Theoretical Physics, Rhein-Westf. Techn. Hochschule, 5100 Aachen, West Germany.
} 
coupling theory for describing the large distance and long time properties of diffusive processes. The result of this analysis is that the presence of disorder in the medium leads to long time correlations that must be taken into account when formulating a macroscopic diffusion equation. The resulting generalized diffusion equation includes memory kernels with slow power law decays, i.e., "long time tails." These occur in the ordinary diffusion term as well as in super-Burnett and higher-order terms. In particular, we will show that the diffusive memory kernel decays like $t^{-(d+2) / 2}$ for long times $t$, were $d$ is the dimensionality of the system. In the context of conductivity problems this result corresponds to a frequency dependence of the conductivity as $\omega \rightarrow 0$.

The theory presented here is applicable to a wide variety of dynamical and stochastic systems. As an important example, we will consider the Lorentz gas ${ }^{(1-3)}$ where the medium is composed of fixed scatterers, usually hard spheres or hard disks. The scatterers are distributed at random, and noninteracting point particles move freely between elastic collisions with the scatterers.

The theory can also be used to describe hopping conductivity. ${ }^{(4,5)}$ Here the medium is a collection of centers from which a moving particle "hops" to neighboring centers with a randomly chosen set of transition rates that govern the jumps to neighboring sites. The "microscopic" motion of the diffusing particle must then be described by a master equation.

Other applications of the theory are to the motion of a particle in a general random potential, of which the Lorentz gas may be considered a special case, and to electrical conduction in a random resistor network. In a subsequent paper we will apply our theory to a variety of examples including the Lorentz gas and several hopping problems. In the present paper we develop the general theory.

We will treat all of these problems within the same framework by taking a coarse-grained view. That is, the description of the detailed structure of the medium is replaced by a simpler one, specified solely by a spatially varying diffusion tensor and a quantity related to the free volume; both of which will be defined below. Spatial variations in these quantities replace the disorder in the original medium. We then treat the resulting fluctuating diffusion equation by the methods of mode-coupling theory. These enable us to obtain the asymptotic long time parts of the memory kernels for the generalized diffusion coefficient and the super-Burnett coefficient.

The motivation of this work stems from the close analogy between transport processes in fluids and transport processes in disordered diffusive media, particularly the Lorentz gas. In the case of fluids, long time tails were first discovered by Alder and Wainwright ${ }^{(6)}$ in their computer simula- 
tions of a hard sphere fluid. They found that the velocity autocorrelation function (VAF) of a $d$-dimensional hard sphere fluid showed an asymptotic long-time decay proportional to $t^{-d / 2}$. Several theoretical explanations of the phenomenon have been given. Kinetic theory can be applied for general fluids at low density ${ }^{(7)}$ and for hard sphere systems at all fluid densities. ${ }^{(7-9)}$ Phenomenological mode-coupling theories can be applied to fluids under general equilibrium conditions. These theories are derived by making certain assumptions about the decay of fluctuations through stages of approximate local equilibrium. ${ }^{(10-13)}$ An alternative to the latter approach is provided by dynamical renormalization group theory, ${ }^{(14-16)}$ which also uses fluctuating hydrodynamic equations as a starting point.

The results of all of these theories are in agreement with each other for both the VAF and time correlation functions related to higher-order transport coefficients, such as the super-Burnett coefficient. The comparison between theory and computer simulations is good at low and moderate densities ${ }^{(17)}$ but the two seem to disagree at high fluid densities. ${ }^{(18,19)}$

For the Lorentz gas the situation is much less satisfactory. Ernst and Weyland ${ }^{(20)}$ showed with the aid of low-density kinetic theory, that for this system the VAF of the moving particle exhibits a long time tail. However, owing to the absence of momentum conservation, it decays asymptotically for long times as $t^{-(d / 2+1)}$, i.e., by one power $t^{-1}$ faster than in a fluid. In computer simulations of the two-dimensional Lorentz gas at low densities Bruin $^{(21)}$ and Lewis and Tjon ${ }^{(22)}$ found a $t^{-2}$ decay of the VAF but obtained a coefficient much larger than that predicted by Ernst and Weyland. More recent computer results, obtained by Alder and Alley, ${ }^{(23,24)}$ indicate that this disagreement is due to the strong density dependence of this coefficient. Extrapolation of their results to zero density yields agreement with the value of Ernst and Weyland at very low density, but the density range over which this agreement persists is inaccessible to the computer. Keyes and Mercer ${ }^{(25)}$ have extended kinetic theory to include excluded volume effects to second order in the density; however these corrections do not bring the theory into agreement with computer experiments.

There have been several efforts to construct theories of the Lorentz gas at high densities based upon a hybrid of mode-coupling ideas and kinetic theory ideas. Götze et al. ${ }^{(26)}$ use the Zwanzig-Mori projection operator formalism to construct a self-consistent kinetic theory which is then solved in a mode-coupling approximation. Keyes and collaborators ${ }^{(27,28)}$ have recently extended this method. While these theories lead to interesting predictions for the behavior of the diffusion coefficient near its percolation threshold they are not in good agreement with the computer experiments for the amplitude of the long time tail. 
The situation sketched above motivated us to develop a mode-coupling theory for the case of tagged particle diffusion in a spatially fluctuating stationary medium. From a formal viewpoint it was somewhat unsatisfac tory to have a well-developed mode-coupling theory based on the fluctuat ing Navier-Stokes equations describing fluid hydrodynamics, but no com parable theory for the simpler diffusion equation. Furthermore, we wantec to develop a simple theory that would permit a comparison with the available computer results for the Lorentz gas and with exact result: obtained for several one-dimensional hopping models. In the end it turnec out that indeed a relatively simple mode-coupling theory describing the type of systems could be derived, but comparison to computer simulation: is not straightforward. The reason is that the coefficient of the long time tai in the VAF involves the mean square of the spatial fluctuations of the diffusion tensor about its average value. In general this quantity is no readily available either from theory or from computer simulations. For $c$ number of one-dimensional hopping models it can be calculated and the validity of our mode-coupling equations is confirmed. For the Lorentz gas in two and higher dimensions no results for these fluctuations are knowr except in the limit of low density of scatterers.

The plan of the paper is as follows: In Section 2 the coarse-grainec description of the random medium is introduced and the parameters entering this description are specified. In Section 3 the mode-coupling equations associated with the coarse-grained description are introduced. In Section 4, relevant correlation functions are analyzed using mode-coupling theory and the long time properties of these correlation functions are presented. The paper is concluded with a discussion of some points that arise in this work, and with an indication of specific applications of the theory which will be discussed in a subsequent paper. ${ }^{(29)}$

\section{QUENCHED AVERAGES AND COARSE-GRAINED DESCRIPTION}

We consider a random medium in a volume $V$ described by a set of parameters which we collectively call $X$. In the case of the Lorentz gas, the set $X$ consists of the number of scatterers $N$ and their positions, $\mathbf{R}_{1} \ldots \mathbf{R}_{N}$. For hopping models, the set $X$ consists of the positions of the lattice sites and the set of transition rates connecting them. Our fundamental assump. tion is that an accurate coarse-grained description of diffusion in the medium can be obtained by replacing the exact microscopic description of the system given by the set $X$ with a more macroscopic description, in which the moving particles are described by a diffusion equation. This fluctuating diffusion equation can be used to calculate the response function (density-density correlation function) from which all transport proper. 
ties of the system can be derived. More precisely, we assume that there are two functions, a spatially varying diffusion tensor $\mathbf{K}(\mathbf{r}, X)$, and a spatially varying free volume fraction $\psi(\mathbf{r}, X)$ relating the local current $\mathbf{J}(\mathbf{r}, t)$ of diffusing particles to the local gradient in the concentration of particles, $c(\mathbf{r}, t)$

$$
\mathbf{J}(\mathbf{r}, t)=-\mathbf{K}(\mathbf{r}, X) \cdot \nabla(c(\mathbf{r}, t) / \psi(\mathbf{r}, X))
$$

Here we assume that the variables $\mathbf{J}$ and $c$ have been coarse grained and leave for later a discussion of the required properties of this coarse graining.

In writing Eq. (2.1), we have made a second assumption; namely, that there are no interactions between the diffusing particles so that $\mathbf{J}$ is linearly related to the gradient of $c$. It follows from this that $\psi$ must be proportional to the equilibrium or stationary concentration of particles since, in the absence of sources and sinks, currents can arise only when there is a deviation from the stationary distribution. First we introduce the concept of quenched averages; next we discuss the coarse-graining method.

We assume that the large volume and long time properties of a single realization of the medium are identical to the average properties of an ensemble of similar realizations. This assumption is less secure than the analogous assumption in classical statistical mechanics since the medium itself is stationary and therefore has no ergodic properties. On the other hand, since the moving particles diffuse they will explore a large volume within the medium and the replacement of a single realization by an ensemble should be safe. What we are going to do is replace the spatial averaging induced by the random motion of the particles by ensemble averaging. It is then mathematically very convenient to employ an ensemble with a distribution $\rho(X)$ to describe the medium so that local fluctuations in the medium are represented by fluctuations from member to member within the ensemble. Now we turn our attention to the definition of $\psi(\mathbf{r}, X)$ that we will use here. As noted above $\psi(\mathbf{r}, X)$ is proportional to the equilibrium, or stationary distribution of particles in the configuration $X$. Then, to define $\psi(\mathbf{r}, X)$ we let $P(\mathbf{r}, X)$ be the equilibrium or stationary probability density for finding a single particle at position $\mathbf{r}$ in configuration $X$. The quantity $\psi(\mathbf{r}, X)$ is defined to be proportional to $P(\mathbf{r}, X)$ by means of the relation

$$
P(\mathbf{r}, X)=\psi(\mathbf{r}, X) / \psi_{0}(X)
$$

where

$$
\psi_{0}(X)=\int d \mathbf{r} \psi(\mathbf{r}, X)
$$

All spatial integrations in this paper extend over the whole volume $V$ of the system. This prescription for $\psi(\mathbf{r}, X)$ is not unique since $\psi$ is defined up to an arbitrary multiplicative function of $X$. To fix this definition for $\psi(\mathbf{r}, X)$ 
we require that $\psi(\mathbf{r}, X)$ have the value unity whenever the moving particle can be considered as "free" at the point $\mathbf{r}$. For Lorentz models this means that $\psi(\mathbf{r}, X)$ is unity whenever the moving particle is not interacting with any scatterers. For stochastic models when the stationary state distributior $P(\mathbf{r}, X)$ is uniform on the lattice sites $\psi(\mathbf{r}, X)$ is set equal to 1 . For thos models where $P(\mathbf{r}, X)$ is not uniform we will set $\psi(\mathbf{r}, X)=\alpha P(\mathbf{r}, X)$ and fix the normalization constant $\psi_{0}(X)$ for our own convenience in each model. Under these circumstances $\psi_{0}(X)$ may be interpreted as the free volume available to a moving particle. The prescription for $\psi(\mathbf{r}, X)$ will perhaps become clearer in the next paper when we consider specific examples.

In order to study time correlation functions we will also need the equilibrium distribution function for the phase of a moving particle in a single configuration $X$. Let $P(\mathbf{r}, \mathbf{v}, X)$ be the probability density for finding a single particle at position $\mathbf{r}$ with velocity $\mathbf{v}$, given that it is in configuration $X$. The single-particle distribution $P(\mathbf{r}, \mathbf{v}, X)$ is then related to $\psi(\mathbf{r}, X)$ by

$$
\int d \mathbf{v} P(\mathbf{r}, \mathbf{v}, X)=\psi(\mathbf{r}, X) / \psi_{0}(X)
$$

From $P(\mathbf{r}, \mathbf{v}, X)$ we can further construct a grand canonical ensemble for the distribution function of many moving particles within a configuration $X$. Since the particles are noninteracting, the distribution of numbers of particles is a Poisson distribution. Let $P_{M}\left(\mathbf{r}_{1} \ldots \mathbf{r}_{M} ; \mathbf{v}_{1} \ldots \mathbf{v}_{M}, X\right)$ be the probability density for finding $M$ particles with phases $\mathbf{r}_{1} \mathbf{v}_{1} \ldots \mathbf{r}_{M} \mathbf{v}_{M}$ within the configuration $X$ :

$$
P_{M}\left(\mathbf{r}_{1} \ldots \mathbf{r}_{M} ; \mathbf{v}_{1} \ldots \mathbf{v}_{M}, X\right)=\frac{1}{M !}\left(\zeta \psi_{0}(X)\right)^{M} e^{-\zeta \psi_{0}(X)} \prod_{j=1}^{M} P\left(\mathbf{r}_{j}, \mathbf{v}_{j}, X\right)
$$

Here $\zeta$ determines the expected number of particles, and for statistical mechanical systems can be identified with the fugacity, $\zeta=\exp \beta \mu$ where $\mu$ is the chemical potential, and $\beta$ the inverse temperature. We denote an average over $P_{M}$ by $\langle\cdots\rangle_{X}$ and an average, first over $P_{M}$ and subsequently over $\rho(X)$ by $\langle\cdots\rangle$. Note that the average $\langle\cdots\rangle_{X}$ is a "quenched" average in the sense that the scatterers are regarded as being fixed with properties specified by $X$. Deviations from these averages are defined by

$$
\begin{gathered}
\hat{f}=f-\langle f\rangle_{X} \\
\delta f=f-\langle f\rangle
\end{gathered}
$$

Next, we turn our attention to the coarse-graining method. In order tc make our fundamental assumption, necessary for the mode-coupling analy. sis of the diffusion equation more explicit, we assume that the spatial correlations in the random medium are sufficiently short ranged (with correlation length $l_{0}$ ). 
Then, suitable coarse graining can be obtained by discarding all the Fourier components with wave number $k$ greater than a cutoff, $k_{c}$ $=2 \pi / L_{c}$, where $L_{c} \gg l_{0}$, while leaving smaller wave vector components unchanged. This determines the length and time scales at which the theory is expected to become accurate.

In this coarse-grained description the local current $\mathbf{J}(\mathbf{r}, t)$ of diffusing particles is assumed to obey the constitutive relation (2.1), which, as we now show, is in agreement with irreversible thermodynamics for those models where a local chemical potential $\mu(\mathbf{r}, t)$ can be defined. First we calculate the quenched average density $\langle c(\mathbf{r})\rangle_{X}$ of moving particles where

$$
c(\mathbf{r})=\sum_{i=1}^{M} \delta\left(\mathbf{r}-\mathbf{r}_{i}\right)
$$

The result follows from (2.5) as

$$
\langle c(\mathbf{r})\rangle_{X}=\zeta \psi(\mathbf{r}, X)
$$

In order to construct irreversible thermodynamics, we must generalize (2.8) to local equilibrium and we imagine that the chemical potential $\mu(\mathbf{r}, t)$ is a slowly varying function of position. We then obtain

$$
\langle c(\mathbf{r}, t)\rangle_{X, \text { local }}=e^{\beta \mu(\mathbf{r}, t)} \psi(\mathbf{r}, X)
$$

If we identify $\langle c(\mathbf{r}, t)\rangle_{X \text {,local }}$ with $c(\mathbf{r}, t)$ in (2.1), we see that the constitutive relation can be written in the standard form of a linear law $\mathbf{J}(\mathbf{r}, t)=$ $-\mathbf{L}(\mathbf{r}, t) \cdot \nabla \mu(\mathbf{r}, t)$ with a local Onsager coefficient $\mathbf{L}(\mathbf{r}, t)$. It is more convenient to use the variables $\mathbf{K}$ and $\psi$ in (2.1) because they depend only on the medium and not on the moving particles.

\section{MODE-COUPLING EQUATIONS}

Our next goal is to obtain a mode-coupling expansion of the fluctuating diffusion equation. To do this we begin by combining the continuity equation with the constitutive relation (2.1), which yields a (spatially) fluctuating diffusion equation

$$
\frac{\partial c(\mathbf{r}, t)}{\partial t}=\boldsymbol{\nabla} \cdot \mathbf{K}(\mathbf{r}, X) \cdot \nabla\left[\frac{c(\mathbf{r}, t)}{\psi(\mathbf{r}, X)}\right]
$$

The equation can be rewritten in terms of fluctuating quantities as

$$
\begin{aligned}
\frac{\partial}{\partial t} \hat{c}(\mathbf{r}, t)= & D \nabla^{2} \hat{c}(\mathbf{r}, t)+\frac{1}{\psi} \boldsymbol{\nabla} \cdot \delta \mathbf{K}(\mathbf{r}, X) \cdot \boldsymbol{\nabla} \hat{c}(\mathbf{r}, t) \\
& -\frac{D}{\psi} \nabla^{2} \hat{c}(\mathbf{r}, t) \delta \psi(\mathbf{r}, X)
\end{aligned}
$$

where $\hat{c}(\mathbf{r}, t)$ is the fluctuation in $c$ about the average, $\langle c\rangle_{X}$, in the 
"quenched" configuration, and

$$
\psi=\langle\psi(\mathbf{r}, X)\rangle
$$

is the average free volume fraction and

$$
D \equiv\left\langle K_{\alpha \alpha}(\mathbf{r}, X)\right\rangle / d \psi
$$

is the bare diffusion coefficient for the system. We use the summation convention in (3.3b). Terms which are second order in the fluctuating quantities have been omitted from (3.2). In Section 4 we show that they d not contribute to the asymptotically dominant time tails.

We next introduce Fourier and Laplace transforms

$$
\begin{aligned}
f_{\mathbf{k}}(t) & =\int d \mathbf{r} e^{-i \mathbf{k} \cdot \mathbf{r}} f(\mathbf{r}, t) \\
f_{\mathbf{k} z} & =\int_{0}^{\infty} d t e^{-z t} f_{\mathbf{k}}(t)
\end{aligned}
$$

The allowed values of $k$ depend on the boundary conditions at the walls 0 : the container. Here periodic boundary conditions will be used, so tha $k_{\alpha}=2 \pi m_{\alpha} / L$ with $\alpha=x, y, \ldots$ and $m_{\alpha}=0, \pm 1, \pm 2 \ldots$. With the aid $\mathrm{o}$ : these transforms the fluctuating diffusion equation can be written in the form

$$
z \hat{c}_{\mathbf{k} z}-\hat{c}_{\mathbf{k}}(0)=-k^{2} D \hat{c}_{\mathbf{k} z}-I \hat{c}_{\mathbf{k} z}
$$

with the operator, $I$, defined by

$$
I f_{\mathbf{k}}=\frac{1}{\psi} \int_{\mathbf{q}}\left(\mathbf{k q}: \delta K_{\mathbf{k}-\mathbf{q}}-k^{2} D \delta \psi_{\mathbf{k}-\mathbf{q}}\right) f_{\mathbf{q}}
$$

and

$$
\int_{\mathbf{q}}()=\frac{1}{V} \sum_{\mathbf{q}}^{k_{c}}() \underset{V \rightarrow \infty}{\longrightarrow} \int^{k_{c}} \frac{d \mathbf{q}}{(2 \pi)^{d}}()
$$

The symbol $\int_{\mathbf{q}}$ indicates a sum over wave numbers which are restricted to being less than a cutoff $k_{c}$, where $k_{c}=2 \pi / L_{c}$ is determined by the coarse-graining length scale. For large volumes the sum approaches ar integral.

Equations (3.2) and (3.5) are formally rather similar to the mode coupling equations found in the theory of fluids ${ }^{(12-16)}$ with two notabls differences. Here the "modes" which are coupled to the dynamical modr $c_{\mathbf{k z}}$ are the Fourier components of the spatially varying quantities $\delta \mathbf{K}$ anc $\delta \psi$. Thus the modes in our theory have no time dependence ${ }^{(28)}$ and ar complicated, nonthermodynamic variables, whereas for fluids the mode: are the hydrodynamic conserved variables whose fluctuations are deter mined thermodynamically. Moreover, the dominant mode coupling effect: in fluids are due to the presence of convective terms in the fluctuatin: equations, while here these terms are absent. 


\section{FLUCTUATION EXPANSIONS OF CORRELATION FUNCTIONS}

The fluctuating diffusion equation will be used to calculate the long time behavior of those time correlation functions that determine the diffusive properties of the system. The most fundamental of these, from which all other correlation functions of interest may be obtained directly, is the intermediate scattering function. This is the Fourier transform of the correlation function of the density of moving particles. It is written as

$$
F(k, t)=\left\langle\hat{c}_{-\mathbf{k}}(0) \hat{c}_{\mathbf{k}}(t)\right\rangle /\left\langle\hat{c}_{-\mathbf{k}}(0) \hat{c}_{\mathbf{k}}(0)\right\rangle
$$

where the average is defined below (2.5). Since the moving particles are noninteracting and Poisson distributed, the average (4.1) over $M$ moving particles can be reduced to an average over a single moving particle, by virtue of the relation $\langle M(M-1)\rangle=\langle M\rangle^{2}$, and $F(\mathbf{k}, t)$ may alternatively be written as the Fourier transform of the self-correlation function

$$
F(k, t)=\left\langle\sum_{i=1}^{M} e^{i \mathbf{k} \cdot \Delta \mathbf{r}_{i}(t)}\right\rangle /\langle M\rangle \equiv\left\langle e^{i \mathbf{k} \cdot \Delta \mathbf{r}(t)}\right\rangle_{s}
$$

Here $\Delta \mathbf{r}(t)=\mathbf{r}(t)-\mathbf{r}(0)$ is the displacement in the time $t$ of a single tagged particle and we have used the relation $\left\langle\hat{c}_{-\mathbf{k}}(0) \hat{c}_{\mathbf{k}}(0)\right\rangle=\langle M\rangle$. In our calculations it is most convenient to use the response function $\left\langle\hat{c}_{-k} \hat{c}_{\mathbf{k} z}\right\rangle$, which is defined as the Laplace transform of $F(k, t)$ and to determine its small $z$ behavior. It is usually expressed in a wave number and frequencydependent diffusion coefficient $U(\mathbf{k}, z)$ :

$$
\left\langle\hat{c}_{-\mathbf{k}} \hat{c}_{\mathbf{k} z}\right\rangle=\left\langle\hat{c}_{-\mathbf{k}} \hat{c}_{\mathbf{k}}\right\rangle /\left\{z+k^{2} U(k, z)\right\}
$$

Here and in the following we use the notation $\hat{c}_{\mathbf{k}}=\hat{c}_{\mathbf{k}}(0)$. For fixed $z$ one may expand $U(k, z)$ in powers of $(i k)^{2}$ as

$$
U(k, z)=U_{0}(z)-k^{2} U_{2}(z)+0\left(k^{4}\right)
$$

where $U_{0}(z)$ and $U_{2}(z)$ are the frequency-dependent diffusion coefficient and (modified) Burnett coefficient, respectively. The diffusion coefficient itself is given by $D=U_{0}(0)$. Odd powers of $(i k)$ in (4.4) vanish due to spatial isotropy of the medium.

The generalized diffusion coefficient $U(k, z)$ can be written as the sum of its bare value $D$ and a wave number and frequency-dependent correction, viz.

$$
U(k, z)=D+\Gamma(k, z)
$$

Our basic assumption was that the small $k$ and $z$ behavior of components of $\hat{c}_{\mathbf{k} z}$ in (4.3) may be calculated from the fluctuating diffusion equation. Thus, combining (3.5) and (4.3), one may express the correction term in 
(4.5) as

$$
\Gamma(k, z)=\frac{1}{k^{2}} \frac{\left\langle\hat{c}_{-\mathbf{k}} I\left\{z+k^{2} D+I\right\}^{-1} \hat{c}_{\mathbf{k}}\right\rangle}{\left\langle\hat{c}_{-\mathbf{k}}\left\{z+k^{2} D+I\right\}^{-1} \hat{c}_{\mathbf{k}}\right\rangle}
$$

The diffusive propagator $\left\{z+k^{2} D+I\right\}^{-1}$ may be expanded in powers of $I$ about the nonfluctuating propagator

$$
G=G(k, z) \equiv\left\{z+k^{2} D\right\}^{-1}
$$

with the result

$$
\left\{z+k^{2} D+I\right\}^{-1}=G-G I G+G I G I G-\cdots
$$

Substitution of the result into Eq. (4.6) yields an expansion for $\Gamma(k, z)$ in powers of $I$ of the form

$$
\begin{aligned}
\Gamma(k, z)= & \frac{z+k^{2} D}{k^{2}} \\
& \times\left\{\frac{\left\langle\hat{c}_{-\mathbf{k}} I G \hat{c}_{\mathbf{k}}\right\rangle}{\left\langle\hat{c}_{-\mathbf{k}} \hat{c}_{\mathbf{k}}\right\rangle}-\frac{\left\langle\hat{c}_{-\mathbf{k}} I G I G \hat{c}_{\mathbf{k}}\right\rangle}{\left\langle\hat{c}_{-\mathbf{k}} \hat{c}_{\mathbf{k}}\right\rangle}+\left(\frac{\left\langle\hat{c}_{-\mathbf{k}} I G \hat{c}_{\mathbf{k}}\right\rangle}{\left\langle\hat{c}_{-\mathbf{k}} \hat{c}_{\mathbf{k}}\right\rangle}\right)^{2}+\cdots\right\}
\end{aligned}
$$

The terms not given explicitly are of cubic and higher order in $I$. The averages occurring in (4.9) contain Fourier-transformed correlation functions $\left\langle\hat{c}_{-\mathbf{k}} \hat{c}_{\mathbf{q}} \delta Y_{\mathbf{k}-1} \cdots\right\rangle$, where $\delta Y_{\mathbf{q}}$ stands for $\delta \psi_{\mathbf{q}}$ or $\delta K_{\mathbf{q}}^{\alpha \beta}$. By first performing the average over the moving particles in a frozen configuration we obtain using (B1) of Appendix B:

$$
\left\langle\hat{c}_{-\mathbf{k}} \hat{c}_{\mathbf{q}}\right\rangle_{X}=\zeta V \psi \delta_{\mathrm{k}, \mathbf{q}}+\zeta \delta \psi_{\mathbf{q}-\mathbf{k}}
$$

Hence $\hat{c}_{-k} \hat{c}_{\mathbf{q}}$ inside an average $\langle\cdots\rangle$ may be replaced by the right-hand side of $(4.10)$. The correlation functions can be decomposed into shortranged cluster functions with a correlation length $l_{0}$ much smaller than the coarse-graining length $L_{c}=2 \pi / k_{c}$ (see Appendix B). Since we are only interested in small wave numbers, we obtain the following results for the Fourier transforms with $|\mathbf{q}|,|\mathbf{k}|,|\mathbf{l}|, \ldots<k_{\mathbf{c}}$ :

$$
\begin{aligned}
\left\langle\hat{c}_{-\mathbf{k}} \hat{c}_{\mathbf{k}}\right\rangle & \cong V \zeta \psi \\
\left\langle\hat{c}_{-\mathbf{k}} \hat{c}_{\mathbf{q}} \delta Y_{\mathbf{k}-\mathbf{q}}\right\rangle & \cong \zeta\left\langle\delta \psi_{0} \delta Y_{0}\right\rangle \\
\left\langle\hat{c}_{-\mathbf{k}} \hat{c}_{\mathbf{q}} \delta Y_{\mathbf{k}-\mathbf{l}} \delta Y_{\mathbf{1 - \mathbf { q }}}\right\rangle & \cong V \zeta \psi \delta_{\mathbf{k}, \mathbf{q}}\left\langle\delta Y_{0} \delta Y_{0}\right\rangle+\zeta\left\langle\delta \psi_{0} \delta Y_{0} \delta Y_{0}\right\rangle
\end{aligned}
$$

An order of magnitude estimate for these averages is

$$
\begin{gathered}
\left\langle\delta \psi_{0} \delta Y_{0}\right\rangle \sim V \psi Y v_{0} \\
\left\langle\delta \psi_{0} \delta Y_{0} \delta Y_{0}\right\rangle \sim V \psi Y^{2} v_{0}^{2}
\end{gathered}
$$


where $v_{0}=l_{0}^{d}$ is a microscopic correlation volume and $\psi=\langle\psi(\mathbf{r}, X)\rangle$ and $Y=\langle Y(\mathbf{r}, X)\rangle$. After this preparation we can calculate the first term in the bracket in (4.9) for $k<k_{c}$ as

$$
\left\langle\hat{c}_{-\mathbf{k}} I G \hat{c}_{\mathbf{k}}\right\rangle /\left\langle\hat{c}_{-\mathbf{k}} \hat{c}_{\mathbf{k}}\right\rangle \cong-\Delta_{\psi} D k^{2} \int_{\mathbf{q}} G(q, z)
$$

where we have introduced

$$
\Delta_{\psi}=\left\langle\left(\delta \psi_{0}\right)^{2}\right\rangle / V \psi^{2}
$$

with the explicit form of $\delta \psi_{0}$ given in (B5).

The term with $\delta K$ vanishes from this expression because its coefficient is odd in q. Similarly we find that the second term in the brackets on the right-hand side of Eq. (4.9) is

$$
\begin{aligned}
\frac{\left\langle\hat{c}_{-\mathbf{k}} I G I G \hat{c}_{\mathbf{k}}\right\rangle}{\left\langle\hat{c}_{-\mathbf{k}} \hat{c}_{\mathbf{k}}\right\rangle} \cong & \Delta_{\psi} D k^{2} G(k, z) \int_{\mathbf{q}} D q^{2} G(q, z) \\
& +\frac{\left\langle\delta K_{0}^{\alpha \beta} \delta K_{0}^{\gamma \beta}\right\rangle}{V \psi^{2}} k_{\alpha} k_{\delta} G(k, z) \int_{\mathbf{q}} q_{\beta} q_{\gamma} G(q, z) \\
& +\frac{\left\langle\left(\delta \psi_{0}\right)^{3}\right\rangle}{V \psi^{3}} D k^{2} \int_{\mathbf{q}} D q^{2} G(q, z) \int_{\mathbf{T}} G(l, z) \\
= & D k^{2} G(k, z)\left[\Delta_{\psi}+\Delta_{K} / d\right] \int_{\mathbf{q}} D q^{2} G(q, z)
\end{aligned}
$$

To derive the last equality in (4.15) we first performed the $q$ integral in the second term on the right-hand side of (4.15) and then used properties of isotropic tensors to write $\left\langle\delta K_{0}^{\alpha \beta} \delta K_{0}^{\gamma \delta}\right\rangle \delta_{\beta \gamma}=\left\langle\delta K_{0}^{\gamma \beta} K_{0}^{\beta \gamma}\right\rangle \delta_{\alpha \delta} / d$ and we introduced

$$
\Delta_{K}=\left\langle\delta K_{0}^{\alpha \beta} \delta K_{0}^{\beta \alpha}\right\rangle / d V(D \psi)^{2}
$$

with the explicit form of $\delta \mathbf{K}_{0}$ given in (B5). In addition, the third-order fluctuation term $\left\langle\left(\delta \psi_{0}\right)^{3}\right\rangle$ in (4.15) may be neglected for small $z$ and sufficiently large coarse-graining volume $V_{c}=\left(2 \pi / k_{c}\right)^{d}=L_{c}^{d}$. This can be seen by using the relation

$$
\int_{\mathbf{q}} D q^{2} G(q, z)=\frac{1}{V_{c}}-z \int_{\mathbf{q}} G(q, z)
$$

and comparing the third-order term with (4.13), showing that it is of relative order $v_{0} / V_{c}$. Substitution of (4.13) and (4.10) into (4.9) yields finally with the help of (4.17) the small $z$ behavior of $\Gamma(k, z)$ in the form

$$
\begin{aligned}
\Gamma(k, z)=-D\{[ & {\left[\Delta_{\psi}+\Delta_{K} / d\right] / V_{c} } \\
& \left.+\left(\Delta_{K} / d\right) z \int_{\mathbf{q}} G(q, z)+d k^{2} \Delta_{\psi} \int_{\mathbf{q}} G(q, z)\right\}
\end{aligned}
$$


where the third term inside brackets in (4.9) has been neglected, as it is o relative order $z$, when compared to the last term kept in (4.18). The resul (4.18) is in the desired form of a $k$ expansion:

$$
\Gamma(k, z)=\Gamma_{0}(z)-k^{2} \Gamma_{2}(z)+\cdots
$$

and the dominant small $z$ singularities in $\Gamma_{0}(z)$ and $\Gamma_{2}(z)$ are given by th second and third term on the right-hand side of this equation, respectively The first term is a $z$-independent contribution to $\Gamma(k, z)$, which togethe with the $z$-independent contributions of the neglected terms, renormalize the bare diffusion coefficient $D$ in (4.5). ${ }^{5}$ The estimates (4.12) show that thi term gives a contribution of order $\left(v_{0} / V_{c}\right)$ to $D$, which can be neglected fo sufficiently large coarse-graining volumes.

We have omitted terms in the mode-coupling operator $I$ that aris from second- and higher-order contributions to the diffusion equation (3.2) The expression for $I$ including both first- and second-order terms is

$$
\begin{aligned}
I f_{k} \equiv[ & \frac{1}{\psi} \int_{\mathbf{q}}\left(\mathbf{k q}: \delta \mathbf{K}_{\mathbf{k}-\mathbf{q}}-k^{2} D \delta \psi_{\mathbf{k}-\mathbf{q}}\right) \\
& \left.+\frac{1}{\psi} \int_{\mathbf{q}} \int_{\mathbf{l}}\left(\mathbf{k} \mathbf{l}: \delta \mathbf{K}_{\mathbf{k}-\mathbf{l}} \delta \psi_{1-\mathbf{q}}+D k^{2} \delta \psi_{\mathbf{k}-\mathbf{1}} \delta \psi_{1-\mathbf{q}}\right)\right] f_{\mathbf{q}}
\end{aligned}
$$

We will denote the second set of terms on the right-hand side as th "bilinear terms," $I^{(2)} f_{k}$, since they are proportional to products of twc fluctuating quantities. The contributions of the bilinear terms to (4.9) ther take the form

$$
\begin{aligned}
& \left\langle\hat{c}_{-\mathbf{k}} I^{(2)} G \hat{c}_{\mathbf{k}}\right\rangle /\left\langle\hat{c}_{-\mathbf{k}} \hat{c}_{\mathbf{k}}\right\rangle \\
& \quad=D k^{2} G(k, z) \Delta_{\psi} / V_{c}+\left\{D_{k}^{2}\left\langle\left(\delta \psi_{0}\right)^{3}\right\rangle / V_{c} V \psi^{3}\right\} \int_{\mathbf{q}} G(q, z)
\end{aligned}
$$

Terms of similar type have already been neglected before since they are o order $v_{0} / V_{c}$ compared to terms that we have kept.

In a similar way one may analyze all higher-order terms in th fluctuation expansion (4.9). Although we have not examined them all, $i$ seems reasonable to conclude from the above analysis that all contribution to the right-hand side of (4.9) omitted in (4.18) are negligible for one of th following reasons: they are smaller than the terms kept by a term of relativi order $v_{0} / V_{c}$, or they are asymptotically less singular in $z$ than the term

\footnotetext{
${ }^{5}$ Denteneer and Ernst ${ }^{(30)}$ have shown explicitly for the example of a one-dimensional hoppin model, where the exact diffusion coefficient and the exact coefficient of the long time tail i known, how higher-order fluctuations renormalize the bare coefficients to yield the exac ones.
} 
kept. Therefore, the terms kept in (4.18) should contain the dominant small $z$ singularities in the expansion coefficients $U_{l}$, with $l=0,2{ }^{6}$

We conclude this section by giving the long time behavior of the generalized transport coefficients and correlation functions. The singularities in $\Gamma_{l}(z)$ and $U_{l}(z)$ are related to the long time behavior of the inverse Laplace transforms. They can be obtained by observing that $G(q, z)$ is the inverse Laplace transform of $\exp \left(-q^{2} D t\right)$. The inverse Laplace transform of the "velocity" correlation function $\phi_{2}(t)$ [see (A4)] becomes for long times:

$$
\phi_{2}(t) \cong-\frac{1}{2} D \Delta_{k}(4 \pi D)^{-d / 2} t^{-(d+2) / 2}
$$

The inverse Laplace transform of $U_{2}(z)$ which is the Burnett correlation function [see (A5)] becomes for $t \rightarrow \infty$

$$
\phi_{4}(t) \cong D^{2} \Delta_{\psi}(4 \pi D t)^{-d / 2}
$$

In a similar way we obtain the following expressions for the long time behavior of the super-Burnett coefficient and modified super-Burnett coefficient defined in (A6):

$$
D_{4}(t) \cong \begin{cases}D_{4}-\frac{D \Delta_{\psi}}{2 \pi(d-2)}(4 \pi D t)^{-d / 2+1} & (d \neq 2) \\ \frac{D \Delta_{\psi}}{4 \pi} \log t & (d=2)\end{cases}
$$

and

$$
B(t) \cong \begin{cases}B-D \frac{2 \Delta_{\psi}+\Delta_{K}}{4 \pi(d-2)}(4 \pi D t)^{-d / 2+1} & (d \neq 2) \\ D \frac{2 \Delta_{\psi}+\Delta_{K}}{8 \pi} \log t & (d=2)\end{cases}
$$

The fluctuation formulas have been defined in (4.14) and (4.16) as

$$
\begin{aligned}
& \Delta_{K}=\frac{1}{V} \frac{1}{(D \psi)^{2}} \frac{1}{d}\left\langle\delta K_{0}^{\alpha \beta} \delta K_{0}^{\beta \alpha}\right\rangle \\
& \Delta_{\psi}=\frac{1}{V} \frac{1}{\psi^{2}}\left\langle\left(\delta \psi_{0}\right)^{2}\right\rangle
\end{aligned}
$$

\footnotetext{
${ }^{6} \mathrm{~W}$. Götze, Ref. 31 , has presented some arguments for the presence of stronger $z$-singularities in $\Gamma(k, z)$ than those found here. However, we have not found evidence of such singularities in our approach, and the relation between the two methods remains to be clarified.
} 
in combination with (B5) of Appendix B:

$$
\begin{aligned}
\delta K_{0}^{\alpha \beta} & =\int d \mathbf{r}\left[K_{\alpha \beta}(\mathbf{r}, X)-\delta_{\alpha \beta} D \psi\right] \\
\delta \psi_{0} & =\int d \mathbf{r}[\psi(\mathbf{r}, X)-\psi]
\end{aligned}
$$

and

$$
\begin{aligned}
\left\langle K_{\alpha \beta}(\mathbf{r}, X)\right\rangle & =\delta_{\alpha \beta} D \psi \\
\langle\psi(\mathbf{r}, X)\rangle & =\psi
\end{aligned}
$$

Finally, if we suppose that the moving particles carry a charge $e$, ws can obtain the frequency-dependent conductivity, $\sigma(\omega)$, from $U_{0}(z)$ using the generalized Einstein relation

$$
\sigma(\omega)=\left(c e^{2} / k_{B} T\right) U_{0}(i \omega)
$$

where $c$ is the density of moving particles. The quantity $U_{0}(z)$ can br obtained from (4.18) and (4.4)-(4.5) for $z \rightarrow 0$ :

$$
U_{0}(z) \cong D+\left(\Delta_{K} / d\right) z D \int \frac{d \mathbf{q}}{(2 \pi)^{d}}\left[z+D q^{2}\right]^{-1}
$$

\section{DISCUSSION}

In this paper we have developed a mode-coupling theory for the lon time properties of diffusion in a random medium. Although our theory i: based upon a phenomenological mode-coupling approach we believe that $i$ gives formally exact results for the coefficients of the long time tails in the frequency-dependent diffusion coefficient and super-Burnett coefficient Our justification for this assertion is that the only features of the phenome nological approach which survive in the final result are the fluctuations ir the bulk quantities $\delta \mathbf{K}_{0}(X)$ and $\delta \psi_{0}(X)$. These features are independent $\mathrm{o}$ the coarse-graining scheme and can be given microscopic definitions.

Our theory is based upon the standard mode-coupling methods user for fluids and the results we obtain are similar to those obtained for fluid: except that in the present case the long time tails decay with one highe power of $t^{-1}$.

Although the underlying phenomenological equations are simpler ir the diffusive case, the results are more complex because the coefficients $o$ the diffusive long time tails are given by fluctuations in nonthermodynamir quantities $\delta \mathbf{K}_{0}$ and $\delta \psi_{0}$. For the long time tails in fluids the amplitudes ar proportional to thermodynamic fluctuations which can be easily evaluated 
On the other hand the fluctuations required in the present theory can rarely be evaluated exactly.

The long time tails of the kind discussed in this paper also appear in the case of fluids, but there they decay with one higher power of $t^{-1}$ than the dominant long time tails so that for very long times they are masked by the ordinary long time tails.

One can see from the calculation presented here that a necessary condition for the validity of our results is that the relative fluctuations $\Delta_{K}$ in (4.16) and $\Delta_{\psi}$ in (4.14) remain small. For $\Delta_{K}$ to remain small the average diffusion coefficient in (4.16) must be nonzero. If this were not true, then the perturbation expansion of $\left\{z+k^{2} D+I\right\}^{-1}$ about the nonfluctuating propagator $\left\{z+k^{2} D\right\}^{-1}$ would not be well defined, and the results quoted for $\phi_{2}, \phi_{4}, B(t)$, and $D_{4}(t)$, in (4.22)-(4.27) would all diverge. This condition makes our method inapplicable to those cases where one knows for other reasons that $D=0$. Examples of such cases are the motion of particles in the overlapping wind-tree model, ${ }^{(32)}$ where "retracing" events lead to a vanishing diffusion coefficient, the motion of particles in the overlapping Lorentz model at high density ${ }^{(23,24)}$ when the trapping of the particles by the scatterers prevents them from diffusing through the system; as well as in some hopping models ${ }^{(4)}$ where diffusion is destroyed by strong disorder. Similar restrictions apply to $\Delta_{\psi}$. For instance in the Lorentz gas with overlapping scatterers there exist regions where the moving particles are trapped, and diffusive regions where the particles are free. The average free volume fraction of the diffusive region as a function of the scatterer density exhibits a phase transition at the percolation density, and consequently $\Delta_{\psi}$ in (4.14) will diverge at the density.

In a subsequent paper ${ }^{(29)}$ we will apply the mode-coupling theory developed here to a number of specific models:

(i) The deterministic Lorentz gas in $d$ dimensions with spherical scatterers, which may or may not overlap each other. The random medium is the system of scatterers, characterized by the density of scatterers, $n .^{(1-3)}$

(ii) The one-dimensional stochastic Lorentz model where the moving particle is reflected by a scatterer with a probability $p$, and transmitted with a probability $(1-p)$. The scatterers are randomly distributed on a line. ${ }^{(33,34)}$

(iii) The one-dimensional waiting time Lorentz model. The random medium is the same as in (ii) and the moving particles jump from one scatterer to its nearest neighbor with stochastically distributed waiting times between jumps. ${ }^{(33,35,36)}$

(iv) The one-dimensional random barrier model, where the moving particle makes instantaneous jumps between neighboring sites on a regular lattice. The jump frequencies are independent random variables. ${ }^{(4,30,36-41)}$ 
(v) Isotropic random jump rate models on regular $d$-dimensiona lattices. The jump rate, which is the same in all allowed directions is : stochastic quantity, with a site-independent probability distribution. ${ }^{(41,42-4 *}$

The method does not seem to be restricted to an analysis of diffusiv properties in media with static disorder using the diffusion equation with . fluctuating diffusion coefficient, but it may be used equally well to discus the elastic properties of such media using the wave equation with fluctual ing elastic constants. In this manner one may determine quantities like th density of states and the inverse localization length. ${ }^{(44-46)}$

\section{ACKNOWLEDGMENTS}

J. R. Dorfman would like to thank E. G. D. Cohen for stimulatin: discussions in the early stages of this work. The authors would like to than] B. J. Alder, W. E. Alley, T. Kirkpatrick, and R. Zwanzig for helpfu discussions and correspondences. M. H. Ernst wishes to express his thank to the Institute for Physical Science and Technology of the University o Maryland for its hospitality in the Fall of 1981 and Spring of 1983. Thi work was supported in part by Grant No. CHE 80-15976 from the Nationa Science Foundation and by a NATO Travel Grant.

\section{NOTE ADDED IN PROOF}

We have been informed that P. Visscher ${ }^{(47)}$ has recently obtainer identical results to ours by a different method.

\section{APPENDIX A}

We show how the transport properties of interest can be calculater from the intermediate scattering function $F(k, t)$ in (4.1) and (4.2). Firs consider the mean square displacement $\left\langle(\Delta x(t))^{2}\right\rangle$, and higher moments o $\Delta x(t)$, where the $x$ direction is chosen parallel to $\mathbf{k}$. The function $F(k, t)$ i the generating function for these moments:

$$
F(k ; t)=1-\frac{1}{2} k^{2}\left\langle[\Delta x(t)]^{2}\right\rangle_{s}+\frac{1}{4 !} k^{4}\left\langle[\Delta x(t)]^{4}\right\rangle_{s}+\cdots
$$

Related to these moments are the time-dependent diffusion coefficient $D(t)$ and super-Burnett coefficient $B(t)$, defined in terms of cumulants of the $x$ displacement according to ${ }^{(47,48)}$

$$
\begin{aligned}
& D(t)=\frac{\partial}{\partial t} \frac{1}{2}\left\langle(\Delta x(t))^{2}\right\rangle_{s} \\
& B(t)=\frac{\partial}{\partial t} \frac{1}{4 !}\left\{\left\langle(\Delta x(t))^{4}\right\rangle_{s}-3\left\langle(\Delta x(t))^{2}\right\rangle_{s}^{2}\right\}
\end{aligned}
$$

The relation between $U_{l}(x)$ in (4.4) and the Laplace transform $\left\langle(\Delta x)_{z}^{l}\right\rangle_{s}$ of 
the moment of displacement $\left\langle(\Delta x(t))^{l}\right\rangle_{s}$ can be obtained by expanding (4.3) in powers of $q^{2}$ using (4.4), and comparing the result with

$$
\begin{aligned}
& \frac{1}{2} z^{2}\left\langle[\Delta x]_{z}^{2}\right\rangle_{s}=U_{0}(z) \\
& \frac{1}{4 !} z^{2}\left\langle[\Delta x]_{z}^{4}\right\rangle_{s}=U_{2}(z)+\frac{1}{z} U_{0}^{2}(z)
\end{aligned}
$$

Defining $\phi_{I+2}(t)$ as the inverse Laplace transform of $U_{l}(z)(l=0,2)$, one sees from (A3) that $\phi_{2}(t)$ is the second derivative of the mean square displacement and therefore (the analog of) the velocity correlation function (VAF):

$$
\phi_{2}(t)=\frac{1}{2}\left(\frac{d}{d t}\right)^{2}\left\langle[\Delta x(t)]^{2}\right\rangle_{s}=\left\langle v_{x}(0) v_{x}(t)\right\rangle_{s}
$$

The last equality is only meaningful if $\Delta x(t)$ can be written as an integral over the $x$ component of a "velocity" $v_{x}(t)$. Similarly, the Burnett correlation $^{(29,30)}$ function $\dot{\phi}_{4}(t)$ is related to the second derivative of the fourth moment, and can be simply expressed in terms of "velocities" by virtue of (A3):

$$
\begin{aligned}
\phi_{4}(t)=\int_{0}^{t} d \tau \int_{\tau}^{t} d \tau^{\prime} & \left\langle\left\langle v_{x} v_{x}(\tau) v_{x}\left(\tau^{\prime}\right) v_{x}(t)\right\rangle_{s}\right. \\
& \left.-\left\langle v_{x} v_{x}(\tau)\right\rangle_{s}\left\langle v_{x}\left(\tau^{\prime}\right) v_{x}(t)\right\rangle_{s}\right\}
\end{aligned}
$$

Similarly it follows from (A2) and (A3) that the time dependent transport coefficients are related to these correlation functions $\phi_{l}(t)$ as

$$
\begin{aligned}
B(t) & =D_{4}(t)-\int_{0}^{t} d \tau(D(t)-D(\tau)) D(t-\tau) \\
D(t) & =\int_{0}^{t} d \tau \phi_{2}(\tau) \\
D_{4}(t) & =\int_{0}^{t} d \tau \phi_{4}(\tau)
\end{aligned}
$$

If $\phi_{2}(t)$ and $\phi_{4}(t)$ decay sufficiently fast for long times, $B(t), D(t)$, and $D_{4}(t)$ will approach, respectively, the ordinary diffusion coefficient $D=$ $U_{0}(0)$, the super-Burnett coefficient, $B$, and the modified super-Burnett coefficient, $D_{4}=U_{2}(0)$.

\section{APPENDIX B}

In this appendix we compute the equal time correlation functions $\left\langle\hat{c}(\mathbf{r}) \hat{c}\left(\mathbf{r}^{\prime}\right) \delta Y\left(\mathbf{r}^{\prime \prime}\right) \cdots\right\rangle$, whose Fourier transforms are given in (4.11). The average is defined below (2.5) and $\hat{c}(\mathbf{r})$ and $\delta Y(\mathbf{r}, X)$ in (2.6). Since $\hat{c}(\mathbf{r})$ and 
$\hat{c}\left(\mathbf{r}^{\prime}\right)$ are the only quantities depending on the phases of the movin: particles, we first perform the average at a frozen configuration $X$, a defined below (2.5). This yields

$$
\left\langle\hat{c}(\mathbf{r}) \hat{c}\left(\mathbf{r}^{\prime}\right)\right\rangle_{X}=\zeta \delta\left(\mathbf{r}-\mathbf{r}^{\prime}\right) \psi(\mathbf{r}, X)
$$

The Fourier transform of this equation yields (4.10) in the body of th paper. Hence, inside the average $\langle\cdots\rangle$ we may replace $\hat{c}(\mathbf{r}) \hat{c}\left(\mathbf{r}^{\prime}\right)$ by th right-hand side of $(\mathrm{B} 1)$ with the result

$$
\begin{aligned}
\left\langle\hat{c}(\mathbf{r}) \hat{c}\left(\mathbf{r}^{\prime}\right)\right\rangle= & \zeta \psi \delta\left(\mathbf{r}-\mathbf{r}^{\prime}\right) \\
\left\langle\hat{c}(\mathbf{r}) \hat{c}\left(\mathbf{r}^{\prime}\right) \delta Y\left(\mathbf{r}^{\prime \prime}\right)\right\rangle= & \zeta \delta\left(\mathbf{r}-\mathbf{r}^{\prime}\right)\left\langle\psi(\mathbf{r}) \delta Y\left(\mathbf{r}^{\prime \prime}\right)\right\rangle \\
= & \zeta \delta\left(\mathbf{r}-\mathbf{r}^{\prime}\right)\left\langle\delta \psi(\mathbf{r}) \delta Y\left(\mathbf{r}^{\prime \prime}\right)\right\rangle \\
\left\langle\hat{c}(\mathbf{r}) \hat{c}\left(\mathbf{r}^{\prime}\right) \delta Y\left(\mathbf{r}^{\prime \prime}\right) \delta Y\left(\mathbf{r}^{\prime \prime \prime}\right)\right\rangle= & \zeta \delta\left(\mathbf{r}-\mathbf{r}^{\prime}\right)\left\langle\psi(\mathbf{r}) \delta Y\left(\mathbf{r}^{\prime \prime}\right) \delta Y\left(\mathbf{r}^{\prime \prime \prime}\right)\right\rangle \\
= & \zeta \psi \delta\left(\mathbf{r}-\mathbf{r}^{\prime}\right)\left\langle\delta Y\left(\mathbf{r}^{\prime \prime}\right) \delta Y\left(\mathbf{r}^{\prime \prime \prime}\right)\right\rangle \\
& +\zeta \delta\left(\mathbf{r}-\mathbf{r}^{\prime}\right)\left\langle\delta \psi(\mathbf{r}) \delta Y\left(\mathbf{r}^{\prime \prime}\right) \delta Y\left(\mathbf{r}^{\prime \prime \prime}\right)\right\rangle
\end{aligned}
$$

where $\psi=\langle\psi(\mathbf{r}, X)\rangle$ is the average free volume fraction. The correlation functions on the right-hand side of these equations refer to local propertie of the static medium. In Section 2 we have explicitly assumed that th spatial correlations in the random medium are short ranged with correla tion length $l_{0}$. Hence, the correlation functions appearing on the last line $o$ (B3) and (B4) have the cluster property, i.e., they vanish whenever an: relative distance exceeds the correlation length $l_{0}$. The Fourier transform (B2)-(B4) with wave numbers $k<k_{c} \ll 2 \pi / l_{0}$ are therefore essentiall: independent of the wave numbers, and may be replaced by their values a zero wave number. This yields (4.11) as used in the body of the paper where

$$
\begin{aligned}
\delta \psi_{0}(X) & =\int d \mathbf{r}(\psi(\mathbf{r}, X)-\psi) \\
\delta K_{0}^{\alpha \beta}(X) & =\int d r\left(K_{\alpha \beta}(\mathbf{r}, X)-\delta_{\alpha \beta} D \psi\right)
\end{aligned}
$$

with $\psi=\langle\psi(\mathbf{r}, X)\rangle$ and $\left\langle K_{\alpha \beta}(\mathbf{r}, X)\right\rangle=\delta_{\alpha \beta} D \psi$ according to (3.3). The esti mates (4.12) also follow immediately.

\section{REFERENCES}

1. H. A. Lorentz, Proc. Amst. Acad. 7:438, 585, 684 (1905).

2. E. H. Hauge, Lecture Notes in Physics, Vol. 31, G. Kirczenow and J. Marro, eds. (Springer Verlag, Berlin, 1974); E. G. D. Cohen, Colloques Internationaux C.N.R.S. No. 236Theories cinetique classiques et relativistes.

3. J. M. J. van Leeuwen and A. Weyland, Physica 36:457 (1967); 38:35 (1968).

4. S. Alexander, J. Bernasconi, W. R. Schneider, and R. Orbach, Rev. Mod. Phys. 53:17! (1981).

5. N. F. Mott and E. A. Davis, Electronic Processes in Non-Crystalline Materials, second ed (Clarendon Press, Oxford, 1979). 
6. B. J. Alder and T. E. Wainwright, Phys. Rev. A1:18 (1970).

7. J. R. Dorfman and E. G. D. Cohen, Phys. Rev. A6:776 (1972).

8. J. R. Dorfman and E. G. D. Cohen, Phys. Rev. A12:292 (1975).

9. P. Resibois and Y. Pomeau, Physica 72:493 (1974).

10. M. H. Ernst, E. H. Hauge, and J. M. J, van Leeuwen, Phys. Rev. A4:2055 (1971); J. Stat. Phys. 15:7 (1967).

11. R. Zwanzig, K. S. J. Nordholm, and W. C. Mitchell, Phys. Rev. A5:2680 (1972).

12. D. Bedeaux and P. Mazur, Physica 70:505 (1974); Physica 76:247 (1974).

13. K. Kawasaki, Ann. Phys. 61:1 (1970).

14. P. C. Hohenberg and B. I. Halperin, Rev. Mod. Phys. 49:435 (1977).

15. S. Ma, Modern Theory of Critical Phenomena (W. A. Benjamin, London, 1976).

16. D. Forster, D. R. Nelson, and M. J. Stephen, Phys. Rev. A16:732 (1977).

17. W. W. Wood, in Fundamental Problems in Statistical Mechanics 111, E. G. D. Cohen, ed. (North-Holland, Amsterdam, 1975).

18. J. J. Erpenbeck and W. W. Wood, J. Stat. Phys. 24:455 (1981).

19. D. J. Evans, Molec. Phys. 37:1745 (1979); J. Stat. Phys. 22:81 (1980); Physica 118A:51 (1983).

20. M. H. Ernst and A. Weyland, Phys. Lett. 34A:39 (1971).

21. C. Bruin, Phys. Rev. Lett. 29:1670 (1972); Physica 72:261 (1974).

22. J. C. Lewis and J. A. Tjon, Phys. Lett. 66A:349 (1978).

23. B. J. Alder and W. E. Alley, J. Stat. Phys. 19:341 (1978); Phys. Rev. Lett. 43:653 (1979); preprint November 1981.

24. W. E. Alley, Ph.D. thesis, Univ. of Calif., Davis (1979). B. J. Alder and W. E. Alley, Perspectives in Statistical Physics, H. J. Raveché, ed. (North-Holland, Amsterdam, 1981).

25. T. Keyes and J. Mercer, Physica 95A:473 (1979).

26. W. Götze, E. Leutheuser and S. Yip, Phys. Rev. A23:2634 (1981); Phys. Rev. A24:100 (1981).

27. A. J. Masters and T. Keyes, Phys. Rev. A25:1010 (1982), A26:2129 (1982); Physica 118A:395 (1983).

28. T. Keyes and J. W. Lyklema, J. Stat. Phys. 27:687 (1982).

29. J. Machta, M. H. Ernst, H. van Beijeren, and J. R. Dorfman, J. Stat. Phys. (to appear).

30. P. J. H. Denteneer and M. H. Ernst, J. Phys. C 16:L961 (1983).

31. W. Götze, Philos. Mag. B43:219 (1981).

32. E. H. Hauge and E. G. D. Cohen, J. Math. Phys. 10:397 (1969).

33. H. van Beijeren, Rev. Mod. Phys. 54:195 (1982); H. van Beijeren and H. Spohn, J. Stat. Phys. 31:231 (1983).

34. P. Grassberger, Physica 103A:558 (1980).

35. M. H. Ernst and H. van Beijeren, J. Stat. Phys. 26:1 (1981).

36. J. Machta, Phys. Rev. B24:5260 (1981).

37. R. Zwanzig, J. Stat. Phys. 28:127 (1982).

38. J. W. Haus, K. W. Kehr, and K. Kitahara, Phys. Rev. B25:4918 (1982).

39. I. Webman and J. Klafter, Phys. Rev. B26:5950 (1982).

40. M. J. Stephen and R. Kariotis, Phys. Rev. B26:2917 (1982).

41. J. Machta, J. Stat. Phys. 30:305 (1983).

42. J. W. Haus, K. W. Kehr, and J. Lyklema, Phys. Rev. B25:2905 (1982).

43. V. Halpern, J. Phys. C 15:L027 (1982).

44. P. J. H. Denteneer and M. H. Ernst, Phys. Rev. $B$ (to appear).

45. S. John, H. Sompolinsky, and M. J. Stephen, Phys. Rev. B 27:5592 (1983).

46. T. M. Nieuwenhuizen, J. Phys. (to appear).

47. P. Visscher, Private communication and Phys. Rev. B (to appear). 